\title{
INDICADORES EPIDEMIOLÓGICOS COMO FERRAMENTAS DE GESTÃO EM SERVIÇOS DE REABILITAÇÃO
}

\author{
Mariana Fioravante Barbosa ${ }^{1}$ \\ Andréa de Jesus Lopes ${ }^{2}$
}

\section{Resumo}

A gestão eficiente de serviços públicos de saúde contribui para a qualidade do atendimento ao usuário, possibilitando adequar programas terapêuticos às demandas da população com deficiência assistida nos centros de reabilitação. Os indicadores epidemiológicos contribuem para a tomada de decisão dos gestores. O objetivo deste estudo foi apresentar a frequência de patologias e deficiências como indicador epidemiológico e a sua utilidade para caracterizar o perfil de usuários e direcionar ações estratégicas de gestão no CER III APAE Pará de Minas. A metodologia incluiu uma coleta de dados, considerando o período de 1 ano ( $1^{\circ}$ semestre 2018 e de 2019), com cálculo de frequência relativa das patologias ou deficiências em relação ao número total de novas admissões nas modalidades de reabilitação física, intelectual, visual e no serviço à pessoa ostomizada. Os resultados apontam que a maior incidência na reabilitação física foi de usuários com Acidente Vascular Encefálico (34\%) e amputações (11\%). Na reabilitação intelectual, destaca-se o Transtorno do Espectro do Autismo (39\%), o Atraso no Desenvolvimento (32\%), bem como a Deficiência Intelectual e Múltipla (ambos $17 \%$ ). Na reabilitação visual foram casos com PC e catarata (ambos 14\%). A principal patologia associada à ostomia foi o câncer (63\%). O aumento significativo da demanda ocorreu na reabilitação intelectual. A baixa rotatividade e o aumento da procura são pontos de alerta que exigirão novas decisões de gestão. Conhecer o perfil de usuários e monitorar a busca por esse serviço permitiu criar estratégias de reorganizar atividades, propondo novos projetos e incluindo formação de grupos de usuários com metas em comum, promovendo a capacitação direcionada da equipe, incluindo a Rede de Cuidados.

Palavras-chave: Gestão. Reabilitação. Indicadores.

\begin{abstract}
Efficient management of public health services contributes to the quality of patient care, enabling the adaptation of therapeutic programs to the demands of the population with disabilities assisted in rehabilitation centers. Epidemiological indicators contribute to the decision making of managers. The objective of this study was to present the frequency of pathologies and disabilities as an epidemiological indicator and their usefulness to characterize the users profile and direct strategic management actions at the CER III APAE Pará de Minas. The methodology included data collection considering the period of 1 year (1st semester 2018 and 2019) with calculation of the relative

\footnotetext{
${ }^{1}$ Especialista em Odontologia para Pacientes com Necessidades Especiais, CER III APAE de Pará de Minas, Pará de Minas, MG, Brasil, saude.parademinas@ apaemg.org.br

${ }^{2}$ Mestre em Ciências da Reabilitação, CER III APAE de Pará de Minas, Pará de Minas, MG, Brasil; Centro Universitário Estácio de Belo Horizonte, andrealopesfisio@gmail.com
}

| Fed. Nac. das Apaes- Fenapaes $\mid$ Brasília/DF | v.9 | nº1

P. $57-71$

| jan./jun.2018| ISSN 2237-4329| 
frequency of pathologies or disabilities in relation to the total number of new admissions in the modalities physical, intellectual, visual rehabilitation and service to the person ostomized. Results indicate that the highest incidence in physical rehabilitation was of users with stroke $(34 \%)$ and amputations (11\%). In intellectual rehabilitation, the highlights were Autism Spectrum Disorder (39\%), Developmental Delay (32\%), Intellectual and Multiple Disability (both 17\%). In visual rehabilitation were cases with $\mathrm{CP}$ and cataract (both 14\%). Main pathology associated with ostomy was cancer $(63 \%)$. Significant increase in demand occurred in intellectual rehabilitation. Low turnover and rising demand are warning points that will require new management decisions. Knowing the user profile and monitoring demand has led to strategies for reorganizing activities, proposing new projects including forming user groups with common goals and promoting targeted team training including care network.

Keywords: Management. Rehabilitation. Indicators.

\section{INTRODUÇÃO}

A Rede de Cuidados à Pessoa com Deficiência no âmbito do Sistema Único de Saúde foi instituída em 2012, a partir da necessidade de ampliar, qualificar e diversificar as estratégias para a atenção às pessoas com deficiência física, auditiva, intelectual, visual, ostomia e múltiplas deficiências. A rede conta com serviços integrados, sendo articulada e efetiva nos diferentes pontos de atenção para atender a esse público, assim como para iniciar as ações de reabilitação e de prevenção precoce de incapacidades (BRASIL, 2012).

As ações e os serviços de reabilitação podem ser ofertados em qualquer ponto de atenção da rede pública de saúde. No entanto, são nos Serviços Especializados em Reabilitação, como Centros Especializados em Reabilitação (CER), que são realizados o diagnóstico, o tratamento, a concessão, a adaptação e a manutenção de tecnologia assistiva, nas modalidades de reabilitação (Auditiva, Física, Intelectual e/ou Visual), constituindo-se como referência para a rede de atenção à saúde no território (BRASIL, 2019).

$\mathrm{O}$ acesso ao serviço de reabilitação está fortemente relacionado à capacidade de acolhimento e resposta às necessidades das pessoas atendidas. Isso requer uma análise e uma definição do perfil epidemiológico dos territórios, dos recursos disponíveis e dos processos de educação permanente das equipes de saúde, para que os resultados do cuidado ofertado possam gerar melhor qualidade de vida e maior grau de autonomia possível à pessoa com deficiência (BRASIL, 2014).

Para que a instituição de saúde possa assegurar maior eficácia técnica, eficiência e efetividade do serviço de reabilitação, o gestor deve estar atento às suas responsabilidades, a saber: planejamento, 


\section{Apøe Ciênciø Artigo}

apaeciencia.org.br/revista

programação, coordenação e supervisão de programas e atividades de saúde, visando a qualidade do atendimento ao usuário, otimização dos recursos e adequação dos programas terapêuticos às demandas da população pensando, não somente do ponto de vista técnico, mas do usuário de saúde (CARVALHO; EDUARDO, 1998). Dessa forma, os indicadores epidemiológicos, tornam-se importantes ferramentas para a tomada de decisão dos gestores, contribuindo para o planejamento estratégico, tático e operacional. O presente estudo objetiva apresentar a frequência de patologias e deficiências como indicador epidemiológico e sua utilidade para caracterizar o perfil de usuários e monitorar mudanças para direcionar ações estratégicas de gestão no CER III APAE Pará de Minas.

\section{DESENVOLVIMENTO}

O CER III APAE de Pará de Minas faz parte da Rede de Cuidados à Saúde da Pessoa com Deficiência, habilitado nas modalidades de Reabilitação Física, Intelectual e Autismo no ano de 2013, com inclusão da modalidade de Reabilitação Visual em 2018. Desde a implantação do CER, a coleta de dados, análise e planejamento das ações são executados pela gestão. A partir de 2018, as informações foram organizadas em quatro grupos de indicadores: produção, resolutividade, epidemiológico e recursos humanos, descritos no Quadro 1, e que fazem parte de uma análise mais abrangente pela gestão. Tais indicadores subsidiam o planejamento, a organização, o controle, a avaliação das ações e dos serviços e a tomada de decisões. A decisão é uma ação tomada com base na análise de informações, corresponde à conversão das informações em ação, entretanto, nem todas instituições as tem de forma organizada para sustentar o processo decisório (BRANCO, 2001).

Quadro 1 - Grupos de indicadores utilizados no CER III APAE Pará de Minas, incluindo indicadores epidemiológicos

\begin{tabular}{|ccc|}
\hline Grupo de Indicador & Descritores & Fonte de Verificação \\
\hline & Número de atendimentos & \\
& Número de usuários atendidos em cada modalidade de reabilitação & Prontuário Eletrônico \\
& Absenteísmo & Business Intelligence (BI) \\
Produção & Controle de Altas e Desligamentos & Planilhas de controle \\
& Tempo de permanência no Serviço de Reabilitação & \\
& Número de Planos Terapêutico Individual (PTI) renovados & \\
& Capacidade Instalada & \\
\hline
\end{tabular}




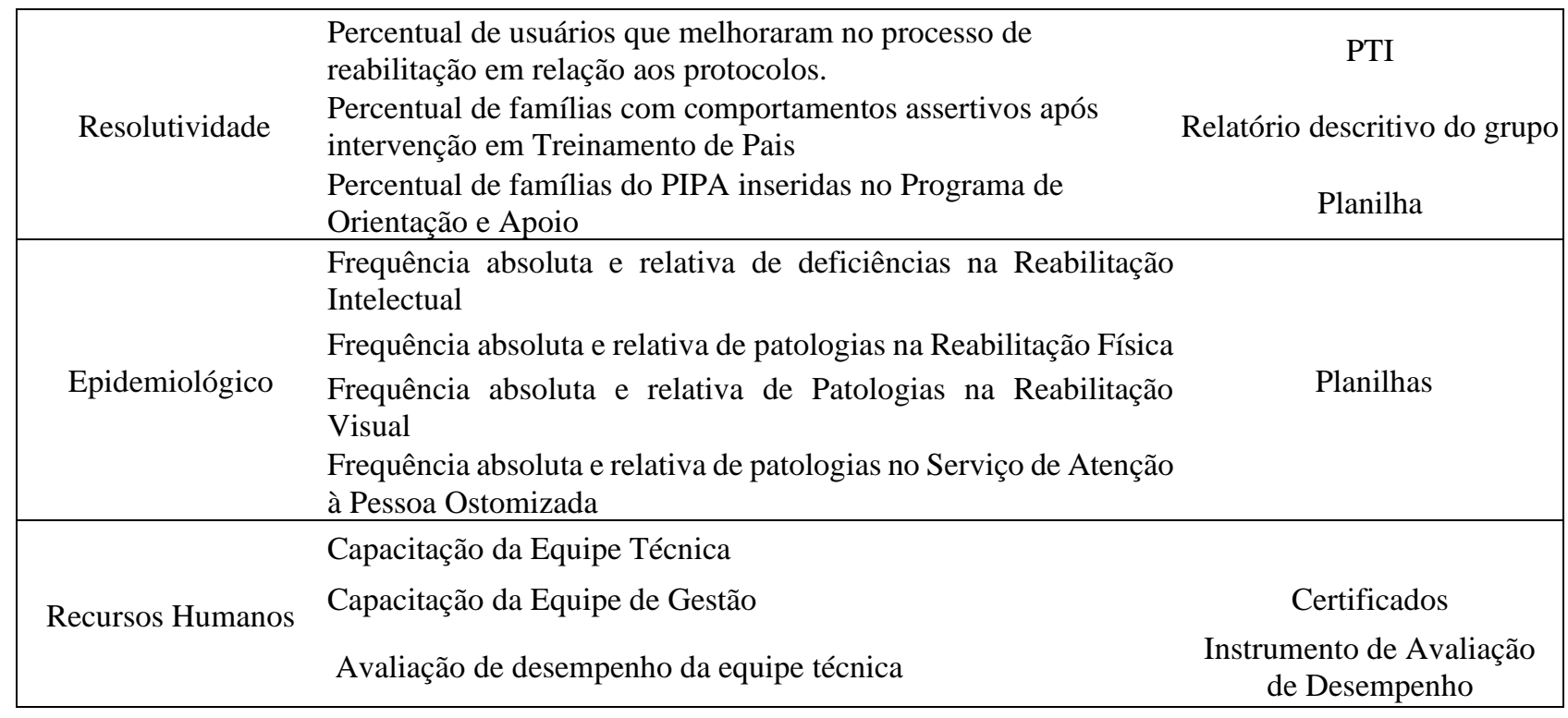

Fonte: Barbosa e Lopes (2019)

As informações foram extraídas de planilhas preenchidas pela equipe técnica após a avaliação diagnóstica inicial na reabilitação física, intelectual, visual e no serviço de atenção à saúde da pessoa ostomizada (SASPO). Semestralmente, os dados são analisados pela gerência de saúde e estabelecidos os planos de ações para o enfrentamento das situações encontradas. No presente estudo, a análise foi restrita aos indicadores epidemiológicos que refletem o perfil das pessoas com deficiência avaliadas no CER III, a fim de caracterizar os usuários admitidos, comparar a ocorrência de patologias e deficiências do $1^{\circ}$ semestre de 2018 com o $1^{\circ}$ semestre de 2019 e monitorar as mudanças para direcionar as estratégias de enfrentamento da gestão, com a finalidade de aprimorar a qualidade dos serviços.

O indicador epidemiológico principal, para este estudo, para caracterizar o perfil dos usuários e as mudanças no tempo foi a frequência de ocorrência relativa, expressa em percentual de admissões em relação à população total admitida no serviço a cada semestre estudado, considerando cada admissão no serviço como uma ocorrência (ROUQUARYOL, 2013).

A Tabela 1 apresenta a frequência relativa das principais patologias e deficiências em admissões no CER III APAE Pará de Minas, de acordo com as modalidades de reabilitação como indicador epidemiológico para caracterizar e conhecer o perfil de usuários admitidos no período. 


\section{Apøe Ciênciø Artigo}

apaeciencia.org.br/revista

Tabela 1- Principais patologias e deficiências admitidas no CER III APAE Pará de Minas e sua frequência relativa, de acordo com as Modalidades de Reabilitação

\begin{tabular}{|c|c|c|c|}
\hline Modalidade de Reabilitação & $\begin{array}{c}\text { Principais } \\
\text { Patologias/ } \\
\text { Deficiências } \\
\text { Diagnosticadas } \\
\end{array}$ & $\begin{array}{l}\text { Frequência relativa } \\
1^{\circ} \text { semestre } 2019\end{array}$ & $\begin{array}{l}\text { Frequência relativa } \\
1^{\circ} \text { semestre } 2018\end{array}$ \\
\hline \multirow{2}{*}{ Reabilitação Física } & AVE & $34 \%$ & $38 \%$ \\
\hline & Amputações & $11 \%$ & $9 \%$ \\
\hline \multirow{2}{*}{ SASPO- Ostomia } & Câncer & $63 \%$ & Manteve \\
\hline & Obstrução Intestinal & $9 \%$ & $6 \%$ \\
\hline \multirow{4}{*}{$\begin{array}{l}\text { Reabilitação Intelectual Avaliação Diagnóstica } \\
\qquad \text { PIPA }^{1}\end{array}$} & TEA & $39 \%$ & \multirow{4}{*}{$\begin{array}{l}\text { Sem dados } \\
\text { comparativos }\end{array}$} \\
\hline & ADNPM & $32 \%$ & \\
\hline & PC & $7 \%$ & \\
\hline & SD & $7 \%$ & \\
\hline \multirow{3}{*}{ Reabilitação Intelectual Serviço Diagnóstico ${ }^{2}$} & TEA & $19,50 \%$ & \multirow{3}{*}{$\begin{array}{l}\text { Sem dados } \\
\text { comparativos }\end{array}$} \\
\hline & $\mathrm{DI}+$ física & $17 \%$ & \\
\hline & DI & $12 \%$ & \\
\hline \multirow{3}{*}{ Reabilitação Intelectual Admissões Gerais* } & TEA & $28 \%$ & $27 \%$ \\
\hline & $\mathrm{DM}$ & $12 \%$ & $13 \%$ \\
\hline & D I & $10 \%$ & $11 \%$ \\
\hline \multirow{2}{*}{ Reabilitação Visual } & $\mathrm{PC}$ & $14 \%$ & \multirow{2}{*}{$\begin{array}{l}\text { Sem dados } \\
\text { comparativos }\end{array}$} \\
\hline & Catarata & $14 \%$ & \\
\hline
\end{tabular}

*Considera a soma das admissões descritas em 1 e 2.

Legenda: AVE: Acidente Vascular Encefálico; SASPO: Serviço de Atenção à Saúde da Pessoa Ostomizada; TEA: Transtorno do Espectro do Autismo; ADNPM: Atraso no Desenvolvimento Neuropsicomotor; PIPA: Programa de Intervenção Precoce Avançada; PC: Paralisia Cerebral; SD: Síndrome de Down; DI: Deficiência intelectual; DM: Deficiência Múltipla.

Fonte: Barbosa e Lopes (2019)

No serviço de Reabilitação Física do CER III APAE Pará de Minas, constata-se que o Acidente Vascular Encefálico (AVE) foi a patologia com maior frequência no período analisado, que corresponde a 34\% da população admitida e diminuição de $4 \%$ de admissões em relação a 2018. As amputações foram a segunda condição de saúde mais frequente, correspondendo a 11\%, com aumento de $2 \%$ de admissões.

De acordo com a Organização Mundial de Saúde (OMS), em todo o mundo, o AVE é a segunda principal causa de morte e a terceira principal causa de incapacidade. Ressalta-se, ainda, que

| Fed. Nac. das Apaes- Fenapaes $\mid$ Brasília/DF | v.9 | nº | P. 57 - 71 | jan./jun.2018 |ISSN 2237-4329| 


\section{Apøe Ciênciø Artigo}

apaeciencia.org.br/revista

nas últimas quatro décadas, a incidência de AVE em países de baixa e média renda mais do que dobrou, enquanto em países de alta renda diminuiu em $42 \%$ a incidência (OMS, 2016).

Diante do alto número de admissões de usuários com AVE, somado ao número de usuários que já se encontravam em atendimento, foi necessário reestruturar o programa de reabilitação física, implementando algumas ações estratégicas, para maior resolutividade, tais como: aumento de sessões de atendimentos em formato intensivo de intervenção, estruturação de uma equipe multidisciplinar direcionada para as demandas desse público com reorganização das agendas dos técnicos de saúde, incremento de recursos terapêuticos incorporando a fisioterapia aquática à abordagem no ginásio de fisioterapia e grupos terapêuticos para desenvolvimento da funcionalidade. Além da reestruturação das estratégias de atendimento no CER III, o apontamento da demanda do público de AVE em fase crônica para a Rede de Atenção Primária resultou em uma organização das ações para o trabalho de acompanhamento pela equipe do Núcleo de Apoio à Saúde da Família (NASF), estabelecendo novos fluxos assistenciais na Rede de Cuidados.

A segunda condição de saúde mais frequentemente admitida no serviço de reabilitação física no CER III APAE Pará de Minas, no $1^{\circ}$ semestre de 2019, são as amputações (11\%) (Tabela 2), sendo distribuída em 50\% de etiologia traumática e 50\% de etiologia vascular. Em relação ao nível de amputação, há uma prevalência maior das amputações transfemorais (50\%), seguida das transtibiais (25\%) e de membros superiores (25\%). Há um maior acometimento no sexo masculino, representando $20 \%$ a mais do que nas mulheres. Com exceção da etiologia, o perfil é semelhante ao encontrado por Garlippe (2014), que avaliou 181 usuários admitidos para reabilitação e identificaram maior prevalência de casos com amputação de origem vascular.

Tabela 2 - Frequência relativa de Amputações, de acordo com a etiologia e o gênero entre os casos admitidos no CER III APAE Pará de Minas no $1^{\circ}$ semestre de 2018 e de 2019

\begin{tabular}{ccccccccc}
\hline Ano & \multicolumn{4}{c}{$\mathbf{1}^{\mathbf{0}}$ semestre 2018 } & \multicolumn{3}{c}{$\mathbf{1}^{\mathbf{o}}$ semestre 2019 } \\
Etiologia & \multicolumn{2}{c}{ Traumática } & \multicolumn{2}{c}{ Vascular } & \multicolumn{2}{c}{ Traumática } & \multicolumn{2}{c}{ Vascular } \\
Nível/Gênero & $\mathbf{M}$ & $\mathbf{F}$ & $\mathbf{M}$ & $\mathbf{F}$ & $\mathbf{M}$ & $\mathbf{F}$ & $\mathbf{M}$ & $\mathbf{F}$ \\
\hline Transtibial & $0 \%$ & $0 \%$ & $17 \%$ & $17 \%$ & $0 \%$ & $0 \%$ & $25 \%$ & $0 \%$ \\
Transfemoral & $17 \%$ & $0 \%$ & $17 \%$ & $17 \%$ & $25 \%$ & $0 \%$ & $25 \%$ & $0 \%$ \\
Membros Superiores & $0 \%$ & $17 \%$ & $0 \%$ & $0 \%$ & $0 \%$ & $25 \%$ & $0 \%$ & $0 \%$ \\
\hline
\end{tabular}

Fonte: Barbosa e Lopes (2019) 


\section{Apøe Ciênci@ Artigo}

apaeciencia.org.br/revista

Dornelas (2007), em estudo epidemiológico analisando 192 indivíduos amputados, verificou que 33,3\% das amputações foram decorrentes de causa externa e os acidentes de transporte, justificando 71,9\% dos casos - a maioria ocorrendo por acidentes em vias urbanas (60,5\%). Desses, quase $50 \%$ foram relacionados às motocicletas. Esses achados reforçam a importância da articulação entre os pontos da Rede de Cuidados para promover campanhas educativas, ações preventivas, educação continuada da equipe técnica e recursos para reabilitar incluindo os dispositivos protéticos dispensados nos centros de reabilitação habilitados para serviços de oficina ortopédica, como é o caso do CER III APAE Pará de Minas.

No SASPO, as principais patologias associadas à ostomia foram câncer (63\%) e obstrução intestinal (9\%). Em relação ao período analisado, a frequência de admissões com diagnóstico de câncer se manteve e houve aumento de $2,8 \%$ de admissões de novos usuários com obstrução intestinal.

Em 2019, na modalidade de Reabilitação Intelectual, a partir da avaliação diagnóstica no Programa de Intervenção Precoce Avançado (PIPA), que avalia crianças de zero a seis anos e 11 meses, o Transtorno do Espectro do Autismo (TEA) foi a condição de maior prevalência com 39\%, seguidas do Atraso de Desenvolvimento Neuropsicomotor (ADNPM) com 32\%, Paralisia Cerebral (PC) e Síndrome de Down (SD), ambas com 7\%. As admissões de usuários acima de 7 anos, pela equipe do serviço de diagnóstico, apresentaram como condições mais frequentes o TEA, com 19,5\%, Deficiência Intelectual, com 12\% e Deficiência múltipla, com 17\%.

Devido à reestruturação do processo de admissão em 2019, não foi possível comparar dados diferenciados entre o PIPA e o Serviço Diagnóstico. Entretanto, ao considerar as duas fontes de admissão de usuários na reabilitação intelectual, as prevalências não se alteraram significativamente. No primeiro semestre de 2018 foi de 13\% para Deficiência múltipla, 27\% para TEA e Deficiência Intelectual $11 \%$ e, em 2019, de 12\% para Deficiência múltipla, 28\% para TEA e Deficiência Intelectual $10 \%$, representando uma variação de $1 \%$ no período, conforme ilustrado na Figura 1. 


\section{Apøe Ciênciø Artigo}

apaeciencia.org.br/revista

Figura 1: Análise comparativa da frequência de admissões gerais na reabilitação intelectual, contemplando todas as faixas etárias, de acordo com o diagnóstico no $1^{\circ}$ semestre de 2018 e de 2019 no CER III APAE Pará de Minas

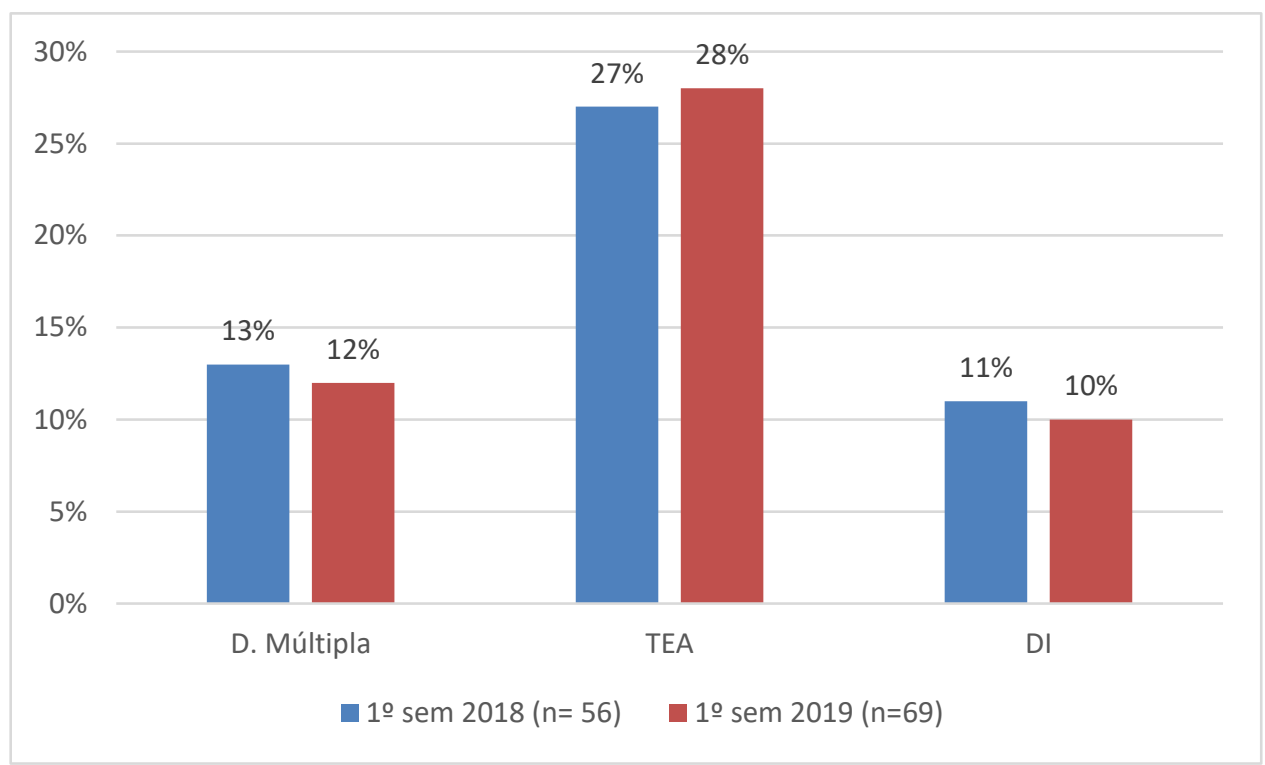

Legenda: D. Múltipla: Deficiência Múltipla; TEA: Transtorno do Espectro do Autismo; DI: Deficiência Intelectual.

Fonte: Barbosa e Lopes (2019)

Em relação ao número total de usuários admitidos no serviço e o número total de atendimentos por semestre, no $1^{\circ}$ semestre de 2018 foram 1.633 usuários e 15.240 atendimentos e no $1^{\circ}$ semestre de 2019, 1.753 usuários e 21.298 atendimentos, com aumento de $7 \%$ de admissões e de $28 \%$ no volume total de atendimentos com equipe técnica de reabilitação atuante nas diferentes modalidades. A admissão de novos usuários acrescida dos usuários que já estão em acompanhamento se torna um indicador de alerta, pois reflete o aumento de demanda para o serviço. Assim, são necessárias decisões importantes da gestão para absorver o volume de pessoas com deficiência da área de abrangência, considerando a capacidade instalada. A relação entre o número de admissões e de alta e desligamentos foi desproporcional no período analisado. Tal dado reflete uma baixa rotatividade relacionada à relativa longa duração de processos de reabilitação, principalmente em crianças e pessoas com deficiência intelectual e múltipla.

Essa demanda teve maior impacto na reabilitação intelectual, com $25 \%$ de admissões, contrastando-se com $75 \%$ de alta e desligamento. Considerando o volume total de usuários no 
período, houve aumento de $4 \%$ de admissões, enquanto as altas e desligamentos totalizaram $43 \%$, justificadas por ações iniciadas no ano anterior com aumento real do número de altas, pois houveram investimentos em novos programas, como as modalidades de treino intensivo, que contribuíram para otimizarem os resultados terapêuticos, principalmente, na Reabilitação Física. Na Reabilitação Intelectual foram realizadas estratégias para estreitarem o vínculo e a participação efetiva das famílias no processo. Além disso, os usuários que não apresentaram adesão e apresentaram faltas excessivas, foram desligados, seguindo o termo pactuado no momento da admissão após os devidos esclarecimentos.

Considerando a complexidade do processo de reabilitação, a permanência prolongada no serviço é uma consequência e a mudança desse cenário depende de avanços científicos e atualizações constantes da equipe técnica atuante na Rede de Cuidados, principalmente nos Centros de Reabilitação.

A literatura define o TEA como uma condição de saúde permanente, de etiologia multifatorial entre fatores genéticos e ambientais e sem cura. Uma definição similar sobre prognóstico é descrita em casos de Deficiência Intelectual. Além disso, tanto o TEA quanto o DI podem coexistir com outras patologias ou deficiências, tornando a reabilitação mais desafiadora. Para exemplificar, o estudo de Schwartzman (2011), discutindo sobre a prevalência e comorbidade relacionada ao TEA, revela a coexistência de deficiência intelectual em aproximadamente $50 \%$ dos casos e, em menor grau, com epilepsia, Síndrome de Down, deficiência auditiva, dentre outras condições patológicas (GUEDES; TADA, 2015).

Essa maior e crescente demanda na modalidade de Reabilitação Intelectual no CER III APAE Pará de Minas acarreta uma gestão de recursos humanos, infraestrutura, projetos e ações para otimizar a admissão e o tratamento no serviço, refletindo em um volume de atendimento superior à meta estipulada para evitar longas listas de espera. Tal fato evidenciado uma demanda para as áreas de psicologia e fonoaudiologia, mas também envolve outros profissionais pelo formato de intervenção multidisciplinar proposto no serviço e para obter maior resolutividade. Guedes e Tada (2015), em uma revisão sistemática sobre as publicações concernentes ao autismo no Brasil, apontam que estudos sobre intervenção são prioritariamente relacionados às intervenções psicoterápicas e fonoaudiológicas, pela necessidade da abordagem de aspectos comportamentais, sociais e de comunicação. Isso reflete a importância da linguagem e do comportamento nas relações sociais como 


\section{Apøe Ciênci@ Artigo}

forma de favorecer a inclusão e a autonomia na vida em sociedade, resultado desejável de todo o programa de reabilitação e com ações para atingir o potencial máximo de cada indivíduo.

Na Reabilitação Visual, devido à habilitação ocorrida em setembro de 2018, foram apresentados apenas os dados relacionados ao primeiro semestre de 2019, que demonstram maior incidência de casos de PC e catarata, ambos com 14\%. Usuários com PC requerem uma abordagem interdisciplinar e vários casos têm alguma outra condição de saúde associada. A oferta de vagas para a Reabilitação Visual representa um avanço para os usuários da rede no município. Em um estudo realizado por Remígio et al. (2006) foram avaliados 274 usuários com deficiência múltipla e identificaram um percentual de 38,7\% de baixa visão. Os autores ressaltaram a necessidade de diagnóstico e tratamento oftalmológico precoce, possibilitando um melhor desenvolvimento global.

Remígio et al. (2006) discutiram que a deficiência visual ou cegueira na infância tem o potencial de comprometer o desenvolvimento motor, cognitivo e da linguagem, pois a visão é para o ser humano, o sentido mais importante para a interação com o ambiente. O percentual ainda foi considerado inferior aos apresentados na literatura, que variam de 45 a $70 \%$, por diferenças no perfil dos participantes dos estudos. Identificou-se o estrabismo como a condição mais prevalente $(25,5 \%)$ e se sabe que essa é uma das alterações oculares mais comuns em crianças com PC. Discutiu-se, também a importância da detecção de deficiência visual de forma precoce em crianças com PC, que é uma demanda então a ser considerada pela gestão do CER, além da atenção aos casos admitidos com catarata.

Ações articuladas e eficientes de toda a Rede de Cuidados de Saúde à Pessoa com Deficiência (PCD) podem ser favorecidas com a troca de informações para alinhar ações de cada ponto de atenção, fortalecendo as estratégias de promoção e prevenção primária e secundária de saúde. Para exemplificar, sabendo que o AVE se destaca no perfil de pessoas com deficiência física admitidas no período, é possível estabelecer estratégias eficazes de prevenção que incluem o direcionamento dos principais fatores modificáveis: hipertensão, lipídios elevados e diabetes.

Os riscos devido aos fatores de estilo de vida também podem ser abordados como tabagismo, baixos níveis de atividade física, dieta não saudável e obesidade abdominal. Essa abordagem poderá contribuir em longo prazo para mudança do perfil de saúde da população da área de abrangência e poderá ter impacto em outras patologias como a doença cardíaca coronária e outras enfermidades vasculares que possuem fatores de risco similares (OMS, 2016). Sendo assim, uma ação direcionada 


\section{Apøe Ciênci@ Artigo}

para mudar uma condição de saúde poderá impactar positivamente em uma teia de condições e favorecer melhoria na saúde e até da qualidade de vida da população.

Dessa forma, a análise dos indicadores pode contribuir para o atendimento integral da saúde dos usuários com deficiência atendidos no Serviço Único de Saúde (SUS).

A clínica e a epidemiologia têm ambas o seu valor e os dados epidemiológicos podem subsidiar saberes e práticas na área clínica e vice-versa (ROUQUAYROL, 2013). O conhecimento do perfil epidemiológico proporciona uma adequação das ações na saúde pública, auxiliando os gestores a melhorarem a assistência clínica. Além disso, os Centros de Reabilitação promovendo atividades eficientes, efetivas e eficazes contribuirão para melhorar a condição de saúde funcional, a qualidade de vida relacionada à saúde e, também, para mudarem o cenário das doenças incapacitantes, corroborando com ações de promoção de saúde e prevenção de agravos na população com deficiência, de forma articulada com outros pontos da Rede de Cuidados à Saúde da PCD.

Dizer que um serviço de reabilitação é a solução para todas as condições de incapacidade e saúde ou de manutenção de funcionalidade e garantia da saúde funcional é uma inverdade. A própria Classificação Internacional de Funcionalidade, Incapacidade e Saúde (CIF), proposta pela Organização Mundial da Saúde, direciona ações diferenciadas, de acordo com os componentes da funcionalidade e as suas complexas interações, conforme o modelo biopsicossocial (FARIAS; BUCHALLA, 2005).

A Rede de Cuidados entende que os Centros de Reabilitação são também considerados como espaço para articulação com outros pontos de atenção do SUS, ou seja, para existir interação entre Atenção Especializada e Atenção Básica, Hospitalar e com Urgência e Emergência. Essa articulação pode ser por troca de saberes e por ações, definição de fluxos do cuidado entre os componentes, por protocolos de ação e procedimentos. Portanto, a Rede de Cuidados à PCD considera o CER como um elo entre os pontos do sistema, incluindo serviços públicos, comunitários e privados de saúde.

Também deve contribuir para a qualificação e a criação em parceria de padrões de cuidados e qualidade mínima para os cuidados às pessoas com deficiência, incluindo para que os profissionais estejam aptos a acolherem as diferenças e humanizarem o atendimento. Essa é uma estratégia política para favorecer para o aprimoramento do SUS, colaborando para a vivência dos seus princípios de universalidade (por ampliar o acesso a serviços de saúde por PCD), de equidade (por tratar diferenças e especificidades e elaborar planos terapêuticos adequados às necessidades e condições apresentadas) 


\section{Apøe Ciênci@ Artigo}

e de integralidade (com grande contribuição dessa sinergia e transversalidade proposta entre os componentes da rede).

Além disso, pode-se contribuir para integrar ações sociais, cooperando para a inclusão e a participação política das PCDs, enfrentamento de rótulos e preconceitos muitas vezes por desconhecimento da sociedade, sabendo que os profissionais de saúde são frutos dela. Cabem ainda ações direcionadas para a formação desses profissionais, colaborando para a mudança de percepção, atitudes e comportamentos frente à deficiência e para promover a discussão entre os segmentos da sociedade acadêmica, prestadora de serviços de saúde e comunidade em geral, para auxiliar com a promoção de melhores condições de saúde e qualidade de vida e autonomia da população com deficiência, bem como aos seus familiares (CAMPOS; SOUZA; MENDES, 2015).

Nesse processo de articular a rede e promover melhoria do cuidado à saúde da PCD é necessário considerar a acessibilidade, nos seus aspectos arquitetônicos e atitudinais. Esse cenário é preocupante. Em estudo realizado por França (2010), avaliando unidades básicas de saúde (UBS) em 41 municípios brasileiros, foi verificada inadequação no acesso em aproximadamente $60 \%$ delas, concluindo que barreiras arquitetônicas interferem negativamente no acesso aos serviços de saúde.

Tais barreiras não se restringem ao interior das UBS, como avaliado por Siqueira et al. (2009), que verificaram ausência de semáforos no entorno em $100 \%$ das unidades avaliadas, calçadas esburacadas (90\%), pisos inadequados (75\%), portas inadequadas, escadas sem corrimão, balcões, bebedouros, banheiros em inadequação com as normas da ABNT. Ações não só relacionadas ao capital humano devem, portanto, fazer parte de um planejamento estratégico para melhorar o cuidado. Vale ressaltar a importância de certificar a acessibilidade física, eliminando os obstáculos, mas, também, envolver a temática sobre acessibilidade atitudinal nas discussões, evitando situações de atitudes inadequadas e preconceituosas da equipe técnica, bem como de apoio e administrativo em toda a Rede de Cuidados.

É necessário, portanto, que os Centros de Reabilitação promovam atividades eficientes, efetivas e eficazes para melhorarem a condição de saúde funcional, qualidade de vida relacionada à saúde e contribuírem para mudarem o cenário das doenças incapacitantes, com ações de promoção de saúde e prevenção de agravos na população com deficiência. A promoção da melhoria na condição biopsicossocial da pessoa com deficiência e sua família deve envolver as esferas municipal, estadual e federal desde a regulação do serviço ao aprimoramento da Rede de Cuidados e criação de políticas que beneficiem essa parcela representativa da população, que pelo censo divulgado pelo IBGE em 


\section{Apøe Ciênciø Artigo}

apaeciencia.org.br/revista

2010 representa 23,4\% dos brasileiros. Outro ponto é a demanda da própria população com deficiência, além da equipe técnica de profissionais de reabilitação, de investimento em pesquisas com levantamento das necessidades de saúde e incapacidades para embasar melhoria contínua na prevenção, promoção, diagnóstico e tratamento e estratégias mais eficientes (TOMAZ et al, 2016).

Por fim, é preciso ressaltar a importância da equipe de reabilitação, sendo ela o principal recurso de atuação junto ao usuário (GUEDES; TADA, 2015). Além disso, é fundamental considerar a família como integrante nessa equipe e principal elo entre o serviço e a comunidade, que pode otimizar o potencial do usuário e favorecer a transferência do ganho de capacidades para o desempenho no contexto de vida real.

\section{CONSIDERAÇÕES FINAIS}

Conhecer o perfil de usuários e monitorar a demanda para os serviços permitiu criar estratégias de reorganizar atividades, formar grupos de usuários com metas em comum, planejar atividades para atender questões relativas à saúde da pessoa com deficiência e promover melhoria contínua na qualidade dos processos de reabilitação conduzidos nos centros especializados.

A baixa rotatividade e o aumento da demanda são pontos de alerta que exigem novas decisões. Em síntese, no último ano, houve o aumento de demanda com percentuais variados, com destaque para a sua elevação na reabilitação intelectual, principalmente admissões de crianças com Transtorno do Espectro do Autismo.

Compartilhar os dados encontrados é de fundamental importância para viabilizar maior conhecimento do perfil de usuários e adequar-se as ações para proporcionar melhor qualidade de atendimento e resolubilidade. Além disso, permite-se fomentar discussões e ações, visando articular a Rede de Cuidados no serviço público voltada para a população com deficiência, assegurando as condições não só para reabilitar, mas também para promover saúde, prevenir agravos, humanizar os atendimentos e poder contribuir para melhorar a sua saúde e a qualidade de vida de toda a população.

\section{REFERÊNCIAS}

BRANCO, Maria Alice Fernandes et al. Informação em saúde como elemento estratégico para a gestão. Gestão municipal de saúde: textos básicos. Brasília: Ministério da Saúde, 2001. p. 163 169. 


\section{Apøe Ciênciø Artigo}

apaeciencia.org.br/revista

BRASIL. Ministério da Saúde. <http://www.saude.gov.br/saude-de-a-z/saude-da-pessoa-comdeficiencia>. Acesso em: 05 out. 2019.

BRASIL. Ministério da Saúde. Portaria GM/MS n ${ }^{\circ} 793$ de 24 de abril de 2012 institui a Rede de Cuidados à Pessoa com Deficiência no âmbito do Sistema Único de Saúde.

BRASIL. Ministério da Saúde. Rede de Cuidados à Pessoa com Deficiência no âmbito do SUS. Instrutivos de reabilitação auditiva, física, intelectual e visual. 2014.

CAMPOS, Mariana Fernandes; SOUZA, Luiz Augusto de Paula; MENDES, Vera Lúcia Ferreira. A Rede de Cuidados do Sistema Único de Saúde à saúde das pessoas com deficiência. InterfaceComunicação, Saúde, Educação, v. 19, p. 207-210, 2015.

CARVALHO, André de Oliveira; EDUARDO, Maria Bernadete de Paula. Sistemas de informação em saúde para municípios. In: Sistemas de informação em saúde para municípios, 1998.

DORNELAS, Lílian de Fátima et al. Amputações por acidentes de transporte: epidemiologia da ocorrência e reabilitação do paciente, 2007.

FARIAS, Norma; BUCHALLA, Cassia Maria. A classificação internacional de funcionalidade, incapacidade e saúde da organização mundial da saúde: conceitos, usos e perspectivas. Revista brasileira de epidemiologia, , v. 8, p. 187-193, 2005.

FRANÇA, Inácia Sátiro Xavier de et al. Violência simbólica no acesso das pessoas com deficiência às unidades básicas de saúde. Revista Brasileira de Enfermagem, v. 63, n. 6, p. 964-970, 2010.

GARLIPPE, Luiz Armando. Estudo epidemiológico dos pacientes com amputação de membros inferiores atendidos no Centro Regional de Reabilitação de Araraquara, Estado de São Paulo, Brasil. 2014. f. Tese (Doutorado) Faculdade, Universidade de São Paulo, São Paulo, 2014.

GUEDES, Nelzira Prestes da Silva; TADA, Iracema Neno Cecílio. A Produção Científica Brasileira sobre Autismo na Psicologia e na Educação. Psicologia: Teoria e Pesquisa, v. 31 n. 3, p. 303-309 Jul-Set 2015

ORGANIZAÇÃO Mundial da Saúde (OMS). Boletim da Organização Mundial da Saúde, 2016.

REMíGIO, Maria Cecília et al. Achados oftalmológicos em pacientes com múltiplas deficiências. Arq Bras Oftalmol, v. 69, n. 6, p. 929-32, 2006.

ROUQUAYROL, Maria Zélia; SILVA, Marcelo Gurgel Carlos da. Epidemiologia \& Saúde. 7. ed. Rio de Janeiro: MedBook, 2013

SIQUEIRA, Fernando Carlos Vinholes et al. Barreiras arquitetônicas a idosos e portadores de deficiência física: um estudo epidemiológico da estrutura física das unidades básicas de saúde em sete estados do Brasil. Ciência \& Saúde Coletiva, Local, v. 14, p. 39-44, 2009.

Fed. Nac. das Apaes- Fenapaes $\mid$ Brasília/DF $\mid$ v.9 | no 1

P. 57 - 71 |jan./jun.2018| ISSN 2237-4329| 


\section{Apøe Ciênci@ Artigo}

apaeciencia.org.br/revista

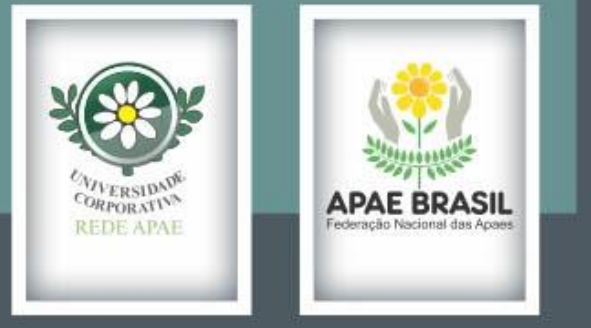

SCHWARTZMAN, José Salomão; ARAÚJO, Ceres Alves (Org.). Transtornos do Espectro do Autismo. São Paulo: Memmon, 2011

TOMAZ, Rodrigo Victor Viana et al. Políticas públicas de saúde para deficientes intelectuais no Brasil: uma revisão integrativa. Ciência \& Saúde Coletiva, v. 21, p. 155-172, 2016. 